\title{
High energy scattering in QCD: from low to high Bjorken $x$
}

\author{
Jamal Jalilian-Marian* \\ Department of Natural Sciences, Baruch College, 17 Lexington Avenue, New York, NY 10010 \\ and \\ CUNY Graduate Center, 365 Fifth Avenue, New York, NY 10016, USA \\ E-mail: jamal.jalilian-marian@baruch.cuny.edu
}

\begin{abstract}
We generalize the Color Class Condensate formalism for particle production in the small Bjorken $x$ kinematics to include contribution of large $x$ gluons. We consider scattering of a quark from the color fields of small and large $x$ gluons of a target proton or nucleus and demonstrate that this leads to spin and angular asymmetries in high energy collisions as well as rapidity loss of the projectile quark.
\end{abstract}

Light Cone 2019 - QCD on the light cone: from hadrons to heavy ions - LC2019

16-20 September 2019

Ecole Polytechnique, Palaiseau, France

${ }^{*}$ Speaker. 


\section{Introduction}

It is a well-known fact that gluon distribution function $x G\left(x, Q^{2}\right)$ of a proton rises very fast with $1 / x$ (equivalently energy) where $x$ is the fraction of the proton energy carried by a gluon and $Q^{2}$ is the resolution at which the proton is probed. This growth of gluons with $1 / x$ must be tamed eventually, as otherwise it would lead to violation of bounds on growth of physical cross sections. A perturbative mechanism which curbs the unlimited of gluon distribution function is the Color Glass Condensate (CGC) formalism [1], an effective theory of QCD at small $x$. Due to the sharp rise of gluon distribution function with $1 / x$, a proton or nucleus becomes a "dense" many-body system of gluons (this density is characterized by the so-called saturation scale $Q_{s}^{2}$ ) where standard perturbative techniques fail due to this high gluon density. The CGC formalism goes around this problem by treating the small $x$ gluons of a proton (or nucleus) collectively; i.e. as a classical background color field radiated by large $x$ partons in the proton.

The simplest implementation of CGC formalism is perhaps in the so called hybrid factorization of scattering and particle production in dilute-dense systems [2], such as a proton-nucleus or forward rapidity proton-proton collision. In this factorization, one consider scattering of a QCDimproved parton model quark or gluon from a dilute projectile scattering on a dense gluonic system standing in for the target proton or nucleus. Due to high gluon density of the target, one needs to consider and re-sum multiple scatterings of the quark on the background color field which is efficiently represented by a Wilson line; a path-ordered exponential of a gluon field. This corresponds to the well-known eikonal approximation in high energy scattering. Quantum effects, i.e. loop corrections are then calculated and included order by order for a given process.

Despite its many successes in describing data on high energy collisions qualitatively and even semi-quantitatively, the CGC formalism has a few shortcomings, perhaps the most significant one being the extreme limit $x \rightarrow 0$ taken in order to justify the approach. This all but ensures that CGC predictions may at best be valid in the kinematic region where the momenta involved are $\mathscr{O}\left(Q_{s}^{2}\right)$ which is not very large. In [3] we proposed to go beyond CGC by including scattering of projectile partons from both small and large $x$ gluons of the target; the small $x$ gluons are still represented as classical color fields from which the projectile scatters many times as before, while the large $x$ gluon of the target is treated perturbatively.

\section{Asymmetries in quark scattering}

In [3] we used spinor helicity methods $[4,5]$ to calculate the helicity amplitudes for scattering of an incoming quark with momentum $p$ and helicity \pm from small and large $x$ gluons (denoted by $S^{-}$and $A^{\mu}$ ) of a target proton (or nucleus). This is the basic process from which one starts to investigate particle production in high energy collisions in the CGC formalism in the forward rapidity region [6]. The momentum of the scattered quark is $q$ (helicity of the final state quark is the same as the initial state quark since helicity is conserved). The light like vector $n^{\mu}$ is defined as $n^{\mu} \equiv\left(n^{+}=0, n^{-}=1, n_{\perp}=0\right)$ such that $n \cdot \mathscr{A}=0$ defines the gauge. There are 3 new (non-eikonal) contributions to the helicity amplitudes; when the initial state and final state quarks scatter from the soft color fields of the target, when the large $x$ gluon interacts with the soft gluons, and when both the final state quark and the large $x$ gluon interact with the soft color fields, labeled as $\mathscr{N}_{123}$ 
respectively. The non-eikonal amplitudes are

$$
\begin{aligned}
\mathscr{N}_{1}^{+, b} & =<\bar{k}^{+}\left|A^{b}(x)\right| k^{+}> \\
\mathscr{N}_{1}^{-, b} & =<\bar{k}^{-}\left|A^{b}(x)\right| k^{-}> \\
\mathscr{N}_{2}^{+, b} & =\frac{1}{(p-\bar{q})^{2}}\left[n \cdot(p-\bar{q})<\bar{q}^{+}\left|A^{b}(x)\right| p^{+}>-<n p>[\bar{q} n](p-\bar{q}) \cdot A^{b}(x)\right] \\
\mathscr{N}_{2}^{-, b} & =\frac{1}{(p-\bar{q})^{2}}\left[n \cdot(p-\bar{q})<\bar{q}^{-}\left|A^{b}(x)\right| p^{-}>-<\bar{q} n>[n p](p-\bar{q}) \cdot A^{b}(x)\right] \\
\mathscr{N}_{3}^{+, b} & =\frac{[\bar{q} \bar{n}]<\bar{n} \bar{p}_{1}>\left[n \cdot\left(p-\bar{p}_{1}\right)<\bar{p}_{1}^{+}\left|A^{b}(x)\right| p^{+}>-<n p>\left[\bar{p}_{1} n\right]\left(p-\bar{p}_{1}\right) \cdot A^{b}(x)\right]}{2 \bar{n} \cdot \bar{p}_{1}\left(p-\bar{p}_{1}\right)^{2}} \\
\mathscr{N}_{3}^{-, b} & =\frac{<\bar{q} \bar{n}>\left[\bar{n} \bar{p}_{1}\right]\left[n \cdot\left(p-\bar{p}_{1}\right)<\bar{p}_{1}^{-}\left|A^{b}(x)\right| p^{-}>-<\bar{p}_{1} n>[n p]\left(p-\bar{p}_{1}\right) \cdot A^{b}(x)\right]}{2 \bar{n} \cdot \bar{p}_{1}\left(p-\bar{p}_{1}\right)^{2}}
\end{aligned}
$$

where $b$ is a color index. Note that

$$
\mathscr{N}_{3}^{+, b}=\frac{[\bar{q} \bar{n}]<\bar{n} \bar{p}_{1}>}{2 \bar{n} \cdot \bar{p}_{1}} \mathscr{N}_{2}^{+, b}\left(\bar{q} \rightarrow \bar{p}_{1}\right)=\mathscr{N}_{2}^{+, b}\left(\bar{q} \rightarrow \bar{p}_{1}\right)
$$

and similarly for $\mathscr{N}_{3}^{-, b}$. These helicity amplitudes (2.1) are related to each other via identities like

$$
\begin{aligned}
& <\bar{k}^{+}\left|X_{b}\right| k^{+}>=<k^{-}\left|A_{b}\right| \bar{k}^{-}> \\
& <\bar{k}^{+}\left|A_{b}\right| k^{+}>=\left(<\bar{k}^{-}\left|A_{b}\right| k^{-}>\right)^{\star}
\end{aligned}
$$

It is straightforward to evaluate these helicity amplitudes which give

$$
\begin{aligned}
\mathscr{N}_{1, b}^{+} & =p^{+} \sqrt{\frac{q^{+}}{p^{+}}}\left\{2 A_{b}^{-}(x)-A_{b}^{i}(x)\left[\frac{k_{1 i}-i \varepsilon_{i j} k_{1}^{j}}{q^{+}}+\frac{k_{i}+i \varepsilon_{i j} k^{j}}{p^{+}}\right]\right\} \\
\mathscr{N}_{2, b}^{+} & =\frac{p^{+}}{q_{\perp}^{2}} \sqrt{\frac{q^{+}}{p^{+}}}\left\{\left(1+\frac{q^{+}}{p^{+}}\right) q_{\perp} \cdot A_{\perp}^{b}(x)+i\left(1-\frac{q^{+}}{p^{+}}\right) \varepsilon^{i j} q_{i} A_{j}^{b}(x)\right\} \\
\mathscr{N}_{3, b}^{+} & =\mathscr{N}_{2}^{+, b}\left(q_{i} \rightarrow p_{1 i}\right) \\
& =\frac{p^{+}}{p_{1 \perp}^{2}} \sqrt{\frac{q+}{p^{+}}}\left\{\left(1+\frac{q^{+}}{p^{+}}\right) p_{1 \perp} \cdot A_{\perp}^{b}(x)+i\left(1-\frac{q^{+}}{p^{+}}\right) \varepsilon^{i j} p_{1 i} A_{j}^{b}(x)\right\}
\end{aligned}
$$

and

$$
\mathscr{N}_{1,2,3}^{-, b}=\left[\mathscr{N}_{1,2,3}^{+, b}\right]^{\star}
$$

To get the scattering cross section one will need to square the amplitude. However, one can see some salient and important features that are already manifest in the amplitude. These non-eikonal amplitudes generate spin asymmetries due to the presence of $i \varepsilon$ terms that will be present in the cross section. Furthermore, coupled with a realistic geometry of the target nucleus, these noneikonal terms will generate angular asymmetries in proton-nucleus and forward proton-proton collisions. We also note that due to exchange of both longitudinal and transverse momenta in the process the incident quark will lose some of its energy (rapidity) which can be related to beam 
rapidity loss in high energy collisions. The next step is to investigate the high transverse momentum of the amplitude. One can then consider loop corrections to this tree-level result due to gluon radiation [7] which would then result in a more general expression for the cross section which would include both collinear factorization and CGC limits in the high and low $p_{t}$ (large and small $x$ ) limits. Such a result would be of enormous benefit specially for processes which are dominated by a very large hard scale, yet receive a large contribution from the small $x$ kinematic region [8].

\section{References}

[1] F. Gelis, E. Iancu, J. Jalilian-Marian and R. Venugopalan, Ann. Rev. Nucl. Part. Sci. 60, 463 (2010) doi:10.1146/annurev.nucl.010909.083629 [arXiv:1002.0333 [hep-ph]]. J. Jalilian-Marian and Y. V. Kovchegov, Prog. Part. Nucl. Phys. 56, 104 (2006) doi:10.1016/j.ppnp.2005.07.002 [hep-ph/0505052].

[2] A. Dumitru and J. Jalilian-Marian, Phys. Rev. Lett. 89, 022301 (2002) doi:10.1103/PhysRevLett.89.022301 [hep-ph/0204028], Phys. Lett. B 547, 15 (2002) doi:10.1016/S0370-2693(02)02709-0 [hep-ph/0111357]; A. Dumitru, A. Hayashigaki and J. Jalilian-Marian, Nucl. Phys. A 765, 464 (2006) doi:10.1016/j.nuclphysa.2005.11.014 [hep-ph/0506308]; J. Jalilian-Marian and Y. V. Kovchegov, Phys. Rev. D 70, 114017 (2004) Erratum: [Phys. Rev. D 71, 079901 (2005)] doi:10.1103/PhysRevD.71.079901, 10.1103/PhysRevD.70.114017 [hep-ph/0405266].

[3] J. Jalilian-Marian, Phys. Rev. D 96, no. 7, 074020 (2017) doi:10.1103/PhysRevD.96.074020 [arXiv:1708.07533 [hep-ph]], Phys. Rev. D 99, no. 1, 014043 (2019) doi:10.1103/PhysRevD.99.014043 [arXiv:1809.04625 [hep-ph]].

[4] L. J. Dixon, hep-ph/9601359.

[5] A. Ayala, M. Hentschinski, J. Jalilian-Marian and M. E. Tejeda-Yeomans, Phys. Lett. B 761, 229 (2016) doi:10.1016/j.physletb.2016.08.035 [arXiv:1604.08526 [hep-ph]]; Nucl. Phys. B 920, 232 (2017) doi:10.1016/j.nuclphysb.2017.03.028 [arXiv:1701.07143 [hep-ph]].

[6] J. Jalilian-Marian, Phys. Rev. C 70, 027902 (2004) doi:10.1103/PhysRevC.70.027902 [nucl-th/0212018]; F. Gelis and J. Jalilian-Marian, Phys. Rev. D 67, 074019 (2003) doi:10.1103/PhysRevD.67.074019 [hep-ph/0211363], Phys. Rev. D 66, 094014 (2002) doi:10.1103/PhysRevD.66.094014 [hep-ph/0208141]; J. Jalilian-Marian, Nucl. Phys. A 753, 307 (2005) doi:10.1016/j.nuclphysa.2005.02.156 [hep-ph/0501222].

[7] A. Ayala, J. Jalilian-Marian, L. D. McLerran and R. Venugopalan, Phys. Rev. D 53, 458 (1996) doi:10.1103/PhysRevD.53.458 [hep-ph/9508302].

[8] E. M. Henley and J. Jalilian-Marian, Phys. Rev. D 73, 094004 (2006) doi:10.1103/PhysRevD.73.094004 [hep-ph/0512220]. 